\title{
Factores de riesgo para el desarrollo de complicaciones posquirúrgicas en pacientes con cáncer renal metastásico tratados con nefrectomía citorreductora
}

\author{
Risk factors for the development of postoperative complications in patients with \\ metastatic kidney cancer treated with cytoreductive nephrectomy
}

\author{
Humberto Hernández-Pañeda, Adrián M. Garza-Gangemi, Héctor H. Manzanilla-Romero, \\ Cristian A. Hernández-Gaytán, Fernando Gabilondo-Navarro y Francisco Rodríguez-Covarrubias* \\ Departamento de Urología, Instituto Nacional de Ciencias Médicas y Nutrición Salvador Zubirán, Ciudad de México, México
}

\begin{abstract}
Resumen
Antecedentes: El papel de la nefrectomía citorreductora como tratamiento del carcinoma de células renales metastásico (CCRm) es controversial debido a su alta complejidad. Objetivo: Identificar factores de riesgo para complicaciones posquirúrgicas en pacientes con CCRm tratados con nefrectomía citorreductora. Método: Estudio retrospectivo, observacional, de 67 pacientes tratados con nefrectomía citorreductora por CCRm. Se registraron las características demográficas, perioperatorias y clinicopatológicas. Las complicaciones posquirúrgicas fueron clasificadas con el sistema Clavien-Dindo (mayores aquellas de grado 3 o superior). Se realizó un análisis de regresión logística binaria para identificar factores de riesgo para complicaciones. Resultados: La edad media fue de 56 años (rango: 37-83), y 58 pacientes (89.7\%) presentaron síntomas, predominando la pérdida de peso (50.8\%). El diámetro tumoral medio fue de $10.8 \mathrm{~cm}$ (rango: $4.6-22.5)$. Un $65 \%$ tuvo complicaciones posquirúrgicas; en el $31.4 \%$ fueron mayores. Los factores de riesgo asociados fueron el sangrado transoperatorio $\geq 500 \mathrm{ml}$ (odds ratio [OR]: 44; intervalo de confianza del 95\% [IC 95\%]: 2.51-789; $p=0.01$ ) y el diámetro tumoral $>10 \mathrm{~cm}$ (OR: 17.9; IC 95\%: 1.2-273; $p=0.04$ ). Conclusiones: La nefrectomía citorreductora es una opción de tratamiento para pacientes estrictamente seleccionados. Nuestra tasa de complicaciones mayores fue del $31.4 \%$. Los factores de riesgo asociados fueron el sangrado transoperatorio y el diámetro tumoral.
\end{abstract}

Palabras clave: Citorreducción. Nefrectomía. Complicaciones posquirúrgicas.

\begin{abstract}
Background: The role of cytoreductive nephrectomy on the treatment of metastatic renal cell carcinoma (mRCC) is controversial due to its high complexity. Objective: To identify risk factors associated to postoperative complications in patients with mRCC after cytoreductive nephrectomy. Method: We conducted a retrospective, observational study in 67 patients who underwent cytoreductive nephrectomy for the management of mRCC. Demographic, perioperative and clinicopathologic characteristics were registered. Surgical complications were classified using the Clavien-Dindo system; major complications were those of grade 3 or higher. We performed a binary logistic regression analysis to identify risk factors associated with
\end{abstract}

\footnotetext{
Correspondencia:

*Francisco Rodríguez-Covarrubias

Vasco de Quiroga 15

Col. Belisario Domínguez, Sección XVI, Del. Tlalpan Fecha de recepción: 07-08-2020

C.P. 14080, Ciudad de México, México Fecha de aceptación: 22-12-2020

E-mail: frodriguez.covarrubias@gmail.com

DOI: $10.24875 /$ CIRU.20000871

Cir Cir. 2021;89(5):632-637

Contents available at PubMed

www.cirugiaycirujanos.com

0009-7411/@ 2020 Academia Mexicana de Cirugía. Publicado por Permanyer. Este es un artículo open access bajo la licencia CC BY-NC-ND (http://creativecommons.org/licenses/by-nc-nd/4.0/).
} 
surgical complications. Results: Mean age was 56 years (37-83). Symptoms were present in 58 patients (89.7\%). Weight loss was the predominant symptom (50.8\%). Mean tumor diameter was $10.8 \mathrm{~cm}(4.6-22.5 \mathrm{~cm})$. The rate of postoperative complications was 65\%; 21 patients (31.4\%) had major complications. Risk factors were estimated blood loss > 500 ml (OR 44.5, Cl 95\% 2.51-789, $p=0.01)$ and tumor diameter > $10 \mathrm{~cm}(O R$ 17.9, Cl 95\% 1.2-273, $p=0.04)$. Conclusions: Cytoreductive nephrectomy is a good option in highly selected patients with mRCC. Our major complication rate was $31.4 \%$. Risk factors associated were blood loss and tumor diameter.

Key words: Cytoreduction surgical procedures. Nephrectomy. Postoperative complications.

\section{Introducción}

El carcinoma de células renales (CCR) representa el $90 \%$ de las neoplasias renales y aproximadamente el $3 \%$ de todas las neoplasias malignas en los adultos $^{1}$. En México, el CCR es la decimoquinta neoplasia en frecuencia, con una tasa de mortalidad del $3.3 \%$. Se estima que la prevalencia entre 2013 y 2018 fue de 8.28 casos por cada 10,000 personas $^{2}$. La enfermedad metastásica se diagnostica en el $20-30 \%$ de los casos y las metástasis pulmonares son las más frecuentes, con una tasa reportada del $45 \%^{3}$.

En décadas recientes, el tratamiento del CCR metastásico $(\mathrm{CCRm})$ ha evolucionado gracias al surgimiento de diversas opciones terapéuticas. En los años 1990, el tratamiento sistémico se basó en la administración de interleucina-2 e interferón alfa $^{3}$. Fue en esta etapa en la que se estableció el potencial benéfico de la nefrectomía citorreductora como parte del tratamiento del $\mathrm{CCRm}^{4}$. La teoría indica que la nefrectomía citorreductora actúa reduciendo la carga tumoral y eliminando la fuente de nuevas metástasis potenciales. Otros mecanismos descritos son la eliminación de factores proangiogénicos y la alteración del microambiente tumoral ${ }^{5,6}$. Posteriormente, la identificación de nuevas dianas terapéuticas permitió desarrollar agentes como los inhibidores del factor de crecimiento endotelial vascular, los inhibidores del factor de crecimiento derivado de plaquetas y algunos otros dirigidos contra la proteína programada de muerte celular ${ }^{3}$, cuyo uso mejoró los resultados oncológicos, poniendo en entredicho el papel de la nefrectomía citorreductora, ya que las nuevas opciones de terapia biológica reducen el espectro de pacientes que se pueden beneficiar del tratamiento quirúrgico agresivo ${ }^{7,8}$. Adicionalmente, la morbilidad asociada a la nefrectomía citorreductora ha obligado a seleccionar cuidadosamente a los pacientes candidatos a este procedimiento ${ }^{9}$.
Por lo tanto, es de vital importancia ponderar las características del paciente y de la neoplasia para apoyar la toma de decisiones en el manejo ${ }^{10}$. Para tales efectos existen modelos pronósticos, como el del International Metastatic RCC Database Consortium (IMDC) y el del Memorial Sloan Kettering Cancer Center, que permiten decidir si los pacientes con CCRm deberán someterse a nefrectomía citorreductora, terapia sistémica o ambas ${ }^{6,11,12}$.

El objetivo del presente estudio fue identificar los factores de riesgo para el desarrollo de complicaciones posteriores a la nefrectomía citorreductora en pacientes con CCRm.

\section{Método}

Este es un estudio retrolectivo, retrospectivo y observacional proveniente de una base de datos de 680 pacientes con CCR tratados quirúrgicamente en nuestra institución, en el cual se analizaron las características demográficas, clínicas, quirúrgicas, histopatológicas y oncológicas de 67 pacientes con CCRm tratados con nefrectomía citorreductora entre marzo de 1980 y diciembre de 2018. Se registraron el índice de masa corporal (IMC) y síntomas tales como hematuria, dolor, masa palpable y pérdida ponderal al momento del diagnóstico de CCRm, así como el sangrado intraoperatorio y las complicaciones posquirúrgicas, catalogadas de acuerdo con la clasificación de Clavien-Dindo ${ }^{13}$. También se registraron otras variables, como la puntuación de la escala de la American Society of Anesthesiologists (ASA) $)^{14}$ y la tasa de filtración glomerular prequirúrgica calculada con la fórmula de la Chronic Kidney Disease Epidemiology Collaboration (CKD-EPI) ${ }^{15}$. El reporte de histopatología utilizó el sistema TNM actualizado en $2018^{16}$ y registró también otras características, como el tipo histológico, el grado de diferenciación (grado nuclear) y los márgenes quirúrgicos, así como la presencia de necrosis, diferenciación rabdoide 0 diferenciación sarcomatoide ${ }^{17}$. Se calcularon las 
puntuaciones de escalas para el estado de desempeño del Eastern Cooperative Oncology Group $(E C O G)^{18}$ y el índice de comorbilidad de Charlson $(\mathrm{CCl})^{19}$.

El análisis estadístico se realizó con SPSS versión 20.0. Para el análisis descriptivo se utilizaron medidas de tendencia central, como la media o la mediana, y para las medidas de dispersión se utilizaron la desviación estándar o el intervalo intercuartílico de acuerdo con la distribución de la variable estudiada. Se analizó la distribución de las variables con la prueba de Shapiro-Wilk. El análisis bivariado se realizó con la prueba t de Student para muestras independientes en variables paramétricas y con la prueba $U$ de Mann-Whitney para variables no paramétricas. Las variables cualitativas se analizaron con la prueba de ji al cuadrado o con la prueba exacta de Fisher. Se analizaron factores de riesgo independientes para complicaciones perioperatorias con regresión logística binaria. Un valor $p \leq 0.05$ se consideró estadísticamente significativo para una distribución de dos colas.

\section{Resultados}

La proporción entre hombres y mujeres fue de 2:1 y la media de edad al diagnóstico fue de 56 años (rango: 37-83). En 58 pacientes (89.7\%) se presentaron síntomas al momento del diagnóstico, siendo la pérdida ponderal el más común (50.8\%), mientras que solo $4(6.3 \%)$ presentaron la tríada clásica de CCR (hematuria, dolor y masa palpable). La media de la tasa de filtración glomerular calculada por la fórmula CKD-EPI fue de $75 \mathrm{ml} / \mathrm{min} / 1.73 \mathrm{~m}^{2}$ (rango: $20-125)$. El $84.1 \%$ tuvo una puntuación $E C O G \leq 1$ y el $96.8 \%$ una puntuación $\geq 5$ en el $\mathrm{CCl}$. La categoría de IMDC clasificó en riesgo intermedio a 46 pacientes $(68.7 \%)$ y en riesgo desfavorable a 21 (31.3\%); no se clasificó ningún paciente como riesgo favorable.

Las características histológicas de los tumores se muestran en la tabla 1. El diámetro tumoral promedio reportado en los estudios de imagen fue de $10.8 \mathrm{~cm}$ (rango: 4.6-22.5). Se identificó CCR de células claras en 61 casos $(92.4 \%)$. Se reportaron 44 tumores (73.3\%) con grados nucleares 3 y 4 , así como 20 con márgenes quirúrgicos positivos (31.2\%). Otras características histológicas, como diferenciación rabdoide y sarcomatoide, estuvieron presentes en $4(6.3 \%)$ y 15 casos (23.8\%), respectivamente. Adicionalmente,
Tabla 1. Características histopatológicas $(n=67)$

\begin{tabular}{lc}
\hline Tipo histológico & \\
Células claras & $61(92.4 \%)$ \\
Otro & $6(7.6 \%)$ \\
Grado nuclear & \\
$1-2$ & $16(26.7 \%)$ \\
$3-4$ & $44(73.3 \%)$ \\
Diferenciación & \\
Rabdoide & $4(6.3 \%)$ \\
Sarcomatoide & $15(23.8 \%)$ \\
Necrosis & \\
Presente & $37(56.9 \%)$ \\
\hline
\end{tabular}

Tabla 2. Complicaciones posquirúrgicas de acuerdo con el sistema Clavien-Dindo $(n=67)$

\begin{tabular}{lc}
\hline Ninguna complicación & $23(34.3 \%)$ \\
I & $1(1.5 \%)$ \\
III & $22(32.8 \%)$ \\
IV & $2(3 \%)$ \\
V & $14(20.9 \%)$ \\
\hline
\end{tabular}

37 piezas quirúrgicas (56.9\%) presentaron algún grado de necrosis.

Las complicaciones posquirúrgicas se describen en la tabla 2. Veintitrés pacientes $(34.3 \%)$ no desarrollaron complicaciones, $23(34.3 \%)$ presentaron complicaciones menores y $19(30.2 \%)$ requirieron cuidados en la unidad de terapia intensiva. En total, 27 pacientes $(45 \%)$ tuvieron un sangrado transoperatorio $>1000 \mathrm{ml}$.

En el análisis univariado (Tabla 3) para determinar los factores de riesgo para cualquier complicación a 30 días se incluyeron el $I M C \geq 30 \mathrm{~kg} / \mathrm{m}^{2}$ (odds ratio [OR]: 0.62; intervalo de confianza del 95\% [IC 95\%]: 0.17-2.26; $p=0.47$ ), la puntuación ASA > 2 (OR: 1.2; IC 95\%: 0.37-3.9; $p=0.76$ ), la hemoglobina prequirúrgica $\leq 12 \mathrm{~g} / \mathrm{dl}$ (OR: 1.73; IC 95\%: 0.62-4.89; $p=0.30$ ), la trombocitopenia prequirúrgica (OR: 2.4; IC 95\%: 0.46-12.4; $p=0.30$ ), el diámetro tumoral $>10 \mathrm{~cm}$ (OR: 8.15; IC 95\%: 1.82-36.5; $p=0.01$ ), la linfadenectomía (OR: 2.5; IC 95\%: 0.77-8.1; $p=0.13$ ) y el sangrado transoperatorio $\geq 500 \mathrm{ml}$ (OR: 16 ; IC 95\%: 3.7-68.9; $p<0.001)$. En el análisis multivariado (Tabla 3), los factores de riesgo independientes para 
Tabla 3. Factores de riesgo para complicaciones a 30 días

\begin{tabular}{lccc}
\hline Variable & OR & IC 95\% & $\mathbf{p}$ \\
\hline Análisis univariado & & & \\
Edad $\geq 65$ años & 0.73 & $0.22-2.38$ & 0.60 \\
Sangrado $\geq 500 \mathrm{ml}$ & 16 & $3.7-68.9$ & $<0.001$ \\
$\mathrm{IMC} \geq 30 \mathrm{~kg} / \mathrm{m}^{2}$ & 0.62 & $0.17-2.26$ & 0.47 \\
Diámetro tumoral > $10 \mathrm{~cm}$ & 8.15 & $1.82-36.5$ & 0.01 \\
Linfadenectomía & 2.5 & $0.77-8.1$ & 0.13 \\
Trombocitopenia prequirúrgica & 2.4 & $0.46-12.4$ & 0.30 \\
ASA $>2$ & 1.2 & $0.37-3.9$ & 0.76 \\
& & & \\
Análisis multivariado & & & \\
Sangrado $\geq 500 \mathrm{ml}$ & 44.5 & $2.51-789$ & 0.01 \\
Diámetro tumoral $>10 \mathrm{~cm}^{*}$ & 17.9 & $1.2-273$ & 0.04 \\
\hline
\end{tabular}

ASA: puntuación en la escala de la American Society of Anesthesiologists; IC 95\%: intervalo de confianza del 95\%; IMC: índice de masa corporal; OR: odds ratio. *Medido en estudios de imagen.

desarrollar complicaciones fueron el sangrado transoperatorio > $500 \mathrm{ml}$ (OR: 44.5; IC 95\%: 2.51-789; $\mathrm{p}=0.01)$ y el diámetro tumoral $>10 \mathrm{~cm}(\mathrm{OR}: 17.9$; IC 95\%: 1.2-273; $p=0.04$ ).

\section{Discusión}

La selección cuidadosa de los pacientes candidatos a nefrectomía citorreductora es esencial, ponderando siempre el riesgo contra el beneficio. Previamente hemos publicado nuestra experiencia con la nefrectomía citorreductora, encontrando que una edad $\geq 65$ años es un factor para el desarrollo de complicaciones y mortalidad perioperatoria en pacientes con $\mathrm{CCRm}^{20}$.

Existe una asociación entre la sintomatología al momento del diagnóstico de CCR y la posibilidad de que el paciente se encuentre en un estadio avanzado de la enfermedad, lo que representa un peor desenlace oncológico ${ }^{21}$.

Acorde con lo anterior, el $89.2 \%$ de nuestra población presentó síntomas, destacando la pérdida ponderal en poco más de la mitad de los casos (50.8\%). Sin embargo, hasta el momento, la asociación con desenlaces quirúrgicos en pacientes sometidos a nefrectomía citorreductora ha sido controversial.

Es importante destacar la heterogeneidad de la función renal en nuestra población. Sin embargo, este aspecto no resultó ser un factor de riesgo relevante para el desarrollo de cualquier complicación posquirúrgica.

El subtipo histológico más común fue el de carcinoma de células claras (92.4\%), lo cual es concordante con las cifras previamente reportadas en nuestro país $^{2}$. En nuestra serie, los grados nucleares 3 y 4 fueron los más frecuentes, lo que, de acuerdo con la literatura, se asocia con enfermedad metastásica hasta en un $79 \%$ de los $\operatorname{casos}^{17}$. El $23.4 \%$ de nuestros pacientes presentaron diferenciación sarcomatoide, hallazgo que también se ha asociado con un peor pronóstico ${ }^{22}$.

Por otra parte, en el $39.1 \%$ de nuestros pacientes se realizó linfadenectomía, ya que cierta evidencia indica que aquellos con sospecha de invasión ganglionar podrían tener mejor supervivencia, sin que aumente el riesgo de complicaciones. Por ello, si esta es técnicamente factible, debe realizarse en los pacientes con sospecha de ganglios positivos ${ }^{8,23}$. Otras series retrospectivas similares han identificado cifras de complicaciones que van del $12 \%$ al $48 \%$, incluyendo un $17 \%$ de complicaciones mayores, lo cual destaca la relevancia de una adecuada selección preoperatoria ${ }^{24-27}$.

Jackson, et al. ${ }^{27}$ estudiaron el tamaño tumoral $\geq 10 \mathrm{~cm}$ como factor de riesgo independiente para un sangrado transoperatorio $\geq 1000 \mathrm{ml}$ con requerimientos de transfusión posteriores $(p=0.021)$, así como mayores complicaciones intraoperatorias. Nuestro estudio obtuvo resultados similares para complicaciones en los 30 días posteriores a la cirugía citorreductora.

O'Malley, et al. ${ }^{24}$ evaluaron la morbilidad asociada a la nefrectomía citorreductora en una cohorte de 65 pacientes recolectados de forma retrospectiva del Roswell Park Cancer Institute, en Nueva York, e identificaron una tasa de complicaciones del $48 \%$, con una tasa de complicaciones mayores del 17\%. Así como en otras series, se ha reportado un menor porcentaje de complicaciones mayores, como se muestra en la tabla 4. Cabe aclarar que, en nuestra población, el $66.6 \%$ de las complicaciones mayores fueron por necesidad de cuidados en la unidad de terapia intensiva.

Nuestro estudio tiene ciertas limitaciones derivadas de su carácter retrospectivo y del número de pacientes incluidos, situaciones que normalmente coinciden en las demás series publicadas. El último aspecto ha afectado incluso a los estudios prospectivos, los cuales se han visto obligados a interrumpir prematuramente la investigación debido a las dificultades en el reclutamiento de pacientes ${ }^{8,9}$.

No obstante, nuestra serie es de las pocas series nacionales existentes, por lo que consideramos que esta información es relevante para la toma de decisiones en nuestro país. Por otra parte, los altos costos de los medicamentos actuales para el tratamiento del 
Cirugía y Cirujanos. 2021;89(5)

Tabla 4. Morbilidad de la nefrectomía citorreductiva

\begin{tabular}{|c|c|c|c|c|c|c|}
\hline Estudio & Diseño, país, años & $\mathbf{N}$ & Edad (años) & $\begin{array}{c}\text { Mortalidad } \\
\text { perioperatoria (\%) }\end{array}$ & $\begin{array}{c}\text { Cualquier } \\
\text { complicación (\%) }\end{array}$ & $\begin{array}{l}\text { Complicaciones } \\
\text { Clavien } \geq 3(\%)\end{array}$ \\
\hline Hernández, et al. (2020) & $\begin{array}{l}\text { Retrospectivo observacional, } \\
\text { México, } \\
\text { 1980-2018 }\end{array}$ & 67 & $\begin{array}{l}\text { Media: } 56 \\
\text { (rango: 37-83) }\end{array}$ & 7.5 & 65.7 & 31.4 \\
\hline Blick, et al. ${ }^{25}$ (2010) & $\begin{array}{l}\text { Cohorte retrospectiva, } \\
\text { multicéntrico, Reino Unido, } \\
\text { 2003-2007 }\end{array}$ & 25 & $\begin{array}{l}\text { Mediana: } 63 \\
\text { (rango: 30-85) }\end{array}$ & 0 & 12 & 4 \\
\hline O'Malley, et al. ${ }^{24}$ (2011) & $\begin{array}{l}\text { Cohorte retrospectiva, } \\
\text { Estados Unidos de América, } \\
\text { 2002-2010 }\end{array}$ & 65 & $\begin{array}{l}\text { Media: } 61 \\
\text { (rango: 42-90) }\end{array}$ & 2 & 48 & 8 \\
\hline Powles, et al. ${ }^{26}$ (2016) & $\begin{array}{l}\text { Ensayo clínico fase 2, } \\
\text { prospectivo, Reino Unido, } \\
\text { 2008-2012 }\end{array}$ & 65 & $\begin{array}{l}\text { Mediana: } 64 \\
\text { (RIC: 56-71) }\end{array}$ & 1 & 22 & 3 \\
\hline Jackson, et al. ${ }^{27}$ (2015) & $\begin{array}{l}\text { Retrospectivo transversal, } \\
\text { Reino Unido, } 2012\end{array}$ & 279 & $\begin{array}{l}\text { Mediana: } 62 \\
\text { (rango: 19-95) }\end{array}$ & 1.8 & 22.6 & 8 \\
\hline
\end{tabular}

RIC: rango intercuartílico.

CCRm hacen que la cirugía citorreductora sea parte de las alternativas de tratamiento en sistemas de salud con limitaciones presupuestarias.

En conclusión, debido a la alta morbilidad quirúrgica y la mayor disponibilidad de nuevas terapias biológicas, el uso de la nefrectomía citorreductora para el tratamiento de los pacientes con CCRm debe basarse en una estricta selección, de manera que solo sean operados aquellos que se verán beneficiados por esta estrategia. En nuestro estudio, el sangrado transoperatorio $\geq 500 \mathrm{ml}$ y el diámetro tumoral $>10 \mathrm{~cm}$ en los estudios de imagen se asociaron con el desarrollo de cualquier complicación en los 30 días posteriores a la cirugía citorreductora. Por estas razones, sugerimos que estos dos factores sean considerados al momento de tomar decisiones sobre el manejo de los pacientes con CCRm.

\section{Responsabilidades éticas}

Protección de personas y animales. Los autores declaran que para esta investigación no se han realizado experimentos en seres humanos ni en animales.

Confidencialidad de los datos. Los autores declaran que han seguido los protocolos de su centro de trabajo sobre la publicación de datos de pacientes.

Derecho a la privacidad y consentimiento informado. Los autores declaran que en este artículo no aparecen datos de pacientes.

\section{Conflicto de intereses}

Los autores declaran no tener conflictos de intereses.

\section{Bibliografía}

1. Capitanio U, Bensalah K, Bex A, Boorjian SA, Bray F, Coleman J, et al. Epidemiology of renal cell carcinoma. Eur Urol. 2019;75:74-84.

2. Sedano-Basilio JE, Mayorga-Gómez E, Garza-Sainz G, Cornejo-Dávila V, Uberetagoyena-Tello de Meneses I, Palmeros-Rodríguez AM, et al. Epidemiología de los tumores genitourinarios en una década. Rev Mex Urol. 2016;76:131-40.

3. Wiechno P, Kucharz J, Sadowska M, Michalski W, Sikora-Kupis B, Jonska-Gmyrek J, et al. Contemporary treatment of metastatic renal cell carcinoma. Med Oncol. 2018;35:135-56.

4. Conti SL, Thomas IC, Hagedorn JC, Chung BI, Chertow GM, Wagner TH, et al. Utilization of cytoreductive nephrectomy and patient survival in the targeted therapy era. Int J Cancer. 2014;134:2245-52.

5. Pindoria N, Raison N, Blecher G, Catterwell R, Dasgupta P. Cytoreductive nephrectomy in the era of targeted therapies: a review. BJU Int. 2017;120:320-8

6. Motzer RJ, Russo P. Cytoreductive nephrectomy - patient selection is key. N Engl J Med. 2018;379:481-2.

7. Bex A, Mulders P, Jewett M, Wagstaff J, Van Thienen JV, Blank CU, et al. Comparison of immediate vs deferred cytoreductive nephrectomy in patients with synchronous metastatic renal cell carcinoma receiving sunitinib: the SURTIME randomized clinical trial. JAMA Oncol. 2019;5:164-70.

8. Méjean A, Ravaud A, Thezenas S, Colas S, Beauval JB, Bensalah K, et al. Sunitinib alone or after nephrectomy in metastatic renal-cell carcinoma. N Engl J Med. 2018;379:417-27.

9. Soares A, Maia MC, Vidigal F, Marques Monteiro FS. Cytoreductive nephrectomy for metastatic renal cell carcinoma: how to apply new evidence in clinical practice. Oncol. 2020:98:1-9.

10. Patel HD, Karam JA, Allaf ME. Surgical management of advanced kidney cancer: the role of cytoreductive nephrectomy and lymphadenectomy. J Clin Oncol. 2018;36:3601-7.

11. Bhindi B, Abel EJ, Albiges L, Bensalah K, Boorjian SA, Daneshmand S, et al. Systematic review of the role of cytoreductive nephrectomy in the targeted therapy era and beyond: an individualized approach to metastatic renal cell carcinoma. Eur Urol. 2019:75:111-28.

12. Castellano-Orozco $M$, Vázquez-Ortega $L$, Camarena-Reynoso $H$, Shuck-Bello C, Leos-Acosta C, Mata-Miranda M, et al. Características clínico-patológicas asociadas a tamaño y metástasis en tumores de células renales. Rev Mex Urol. 2008:68:207-14. 
13. Dindo D, Demartines N, Clavien PA. Classification of surgical complications: a new proposal with evaluation in a cohort of 6336 patients and results of a survey. Ann Surg. 2004;240:205-13.

14. Abouleish A, Leib M, Cohen N. ASA provides examples to each ASA physical status class. ASA Monit. 2015;38-9.

15. Levey AS, Bosch JP, Lewis JB, Greene T. Annals of Internal Medicine, Philadelphia. JAMA J Am Med Assoc. 1962;182:217.

16. Taneja K, Williamson SR. Updates in pathologic staging and histologic grading of renal cell carcinoma. Surg Pathol Clin. 2018;11:797-812.

17. Fuhrman SA, Lasky LC, Limas C. Prognostic significance of morphologic parameters in renal cell carcinoma. Am J Surg Pathol. 1982;6:655-63.

18. Oken M, Creech R, Tormey D, Horton J, Davis T, McFadden E, et al. Toxicity and response criteria of the Eastern Cooperative Oncology Group. Am J Clin Oncol. 1982;5:649-56.

19. Charlson M, Pompei P, Ales K, MacKenszie C. A new method of classifying prognostic in longitudinal studies: development. J Chronic Dis. 1987;40:373-83.

20. Cruz-Ruiz J, Cayetano-Alcaráz AA, Chamlati-Cuello JM, Rodríguez-Covarrubias $\mathrm{F}$. Complicaciones perioperatorias asociadas a nefrectomía en pacientes con cáncer renal metastásico. Gac Mex Oncol. 2019;18:48-52.
21. Campbell SC, Lane BR. Malignant renal tumors. En: Wein A, Kavoussi L, Partin A, Peters C, editores. Campbell-Walsh Urology. $11^{\text {th }}$ ed. Philadelphia: Elsevier; 2016. p. 1320-1.

22. Silagy AW, Mano R, Blum KA, Dinatale RG, Marcon J, Tickoo SK, et al. The role of cytoreductive nephrectomy for sarcomatoid renal cell carcinoma: a 29-year institutional experience. Urology. 2019;136:169-75.

23. Pantuck AJ, Zisman A, Dorey F, Chao DH, Han KR, Said J, et al. Renal cell carcinoma with retroperitoneal lymph nodes: role of lymph node dissection. J Urol. 2003;169:2076-83.

24. O'Malley RL, Brewer KA, Hayn MH, Kim HL, Underwood W, Pili R, et al. Impact of cytoreductive nephrectomy on eligibility for systemic treatment and effects on survival: are surgical complications or disease related factors responsible? Urology. 2011;78:595-600.

25. Blick C, Bott S, Muneer A, Barber NJ, Hindley R, Eden C, et al. Laparoscopic cytoreductive nephrectomy. J Endourol. 2010;24:1451-5.

26. Powles T, Sarwar N, Stockdale A, Sarker SJ, Boleti E, Protheroe A, et al. Safety and efficacy of pazopanib therapy prior to planned nephrectomy in metastatic clear cell renal cancer. JAMA Oncol. 2016;2:1303-9.

27. Jackson BL, Fowler S, Williams ST. Perioperative outcomes of cytoreductive nephrectomy in the UK in 2012. BJU Int. 2015;116:905-10. 